\title{
Liraglutide attenuates the osteoblastic differentiation of MC3T3-E1 cells by modulating AMPK/mTOR signaling
}

\author{
XIONG-KE HU, XIN-HUA YIN, HONG-QI ZHANG, CHAO-FENG GUO and MING-XING TANG
}

Department of Spine Surgery, Xiangya Hospital of Central South University, Changsha, Hunan 410013, P.R. China

Received October 7, 2015; Accepted July 29, 2016

DOI: $10.3892 / \mathrm{mmr} .2016 .5729$

\begin{abstract}
Liraglutide, a synthetic analogue of glucagon-like peptide-1, is utilized in the treatment of type 2 diabetes and obesity. Liraglutide has been previously demonstrated to prevent osteoblastic differentiation of human vascular smooth muscle cells, resulting in the slowing of arterial calcification, however, its effect on bone formation remains unclear. The present study investigated the effect of liraglutide on osteoblastic differentiation using Alizarin Red S staining, and examined the molecular mechanisms underlying the regulatory effect by western blot analysis. The present study demonstrated that protein expression levels of phosphorylated adenosine monophosphate-activated protein kinase ( $\mathrm{p}$-AMPK) were downregulated in MC3T3-E1 cells during osteoblastic differentiation in commercial osteogenic differentiation medium, whereas protein expression levels of transforming growth factor- $\beta$ (TGF- $\beta$ ) and phosphorylated mammalian target of rapamycin (p-mTOR) increased. Liraglutide was subsequently demonstrated to dose-dependently attenuate the osteoblastic differentiation of MC3T3-E1 cells, to upregulate p-AMPK, and downregulate $\mathrm{p}-\mathrm{mTOR}$ and TGF- $\beta$ protein expression levels. Treatment with an AMPK-specific inhibitor, Compound C, eradicated the effect of liraglutide on osteoblastic differentiation, and p-mTOR and TGF- $\beta$ downregulation. An mTOR activator, MHY1485, also abolished the inhibitory effect of liraglutide on osteoblastic differentiation, and resulted in p-mTOR and TGF- $\beta$ downregulation, but did not attenuate the liraglutide-induced increase in p-AMPK protein expression levels. The results of the present study demonstrate that liraglutide attenuates osteoblastic differentiation of MC3T3-E1 cells via modulation of AMPK/mTOR signaling. The present study revealed a novel function of liraglutide, which contributes to the understanding of its pharmacological and physiological effects in clinical settings.
\end{abstract}

Correspondence to: Professor Hong-Qi Zhang, Department of Spine Surgery, Xiangya Hospital of Central South University, 87 Xiangya Road, Changsha, Hunan 410013, P.R. China

E-mail: zhq9006@163.com

Key words: liraglutide, osteoblastic differentiation, AMPK

\section{Introduction}

Liraglutide is a synthetic analogue of glucagon-like peptide-1 (GLP-1) that is commonly used in the management of type 2 diabetes (T2D) and obesity. Liraglutide shares $97 \%$ sequence identity with native human GLP-1 and induces similar biological effects, including enhanced glucose-dependent insulin secretion, accelerated insulin $\beta$-cell proliferation and inhibition of $\beta$-cell apoptosis (1). A recent study demonstrated that liraglutide aids the prevention of T2D complications, such as diabetic nephropathy, caused by exposure to high glucose concentrations (2). The plasma half-life of liraglutide is $\sim 13 \mathrm{~h}$, compared with the $1.5-2.1 \mathrm{~min}$ half-life of native GLP-1, increasing its bioavailability compared with native GLP-1 (3). Liraglutide is also effective in assisting with weight loss, as it delays gastric emptying, slows carbohydrate absorption and increases satiety (3). Liraglutide was previously considered to have minor side effects, and received marketing authorization from medical agencies in Europe, Canada, Japan, and the USA, however, the drug has now been associated with the development of hypoglycemia, acute pancreatitis and thyroid carcinoma (4). Thus, the future clinical application of liraglutide requires comprehensive consideration.

Bone formation is largely dependent on the transformation (differentiation) of osteoblasts into mature osteocytes, which make up $>90 \%$ of all bone cells in the adult skeleton (5). Osteoblastic differentiation is a carefully orchestrated process, and is tightly regulated by signaling pathways, including adenosine monophosphate-activated protein kinase (AMPK)-mediated signaling. AMPK stimulates the proliferation, mineralization and differentiation of osteoblastic MC3T3-E1 cells, when activated by the AMP analog 5-aminoimidazole-4-carboxamide ribonucleotide, the adiponectin adipokine and the lipid-lowering agent, Bezafibrate $(6,7)$. However, AMPK activated by the adipokine $\mathrm{Clq} /$ tumor necrosis factor-related protein 3 , is reported to repress the differentiation of osteoblastic MC3T3-E1 cells (8). Additionally, Wei et al (9) observed that glucose uptake favors osteoblast differentiation by inhibiting the functions of AMPK in vivo and in vitro. The reason for the discrepancy of AMPK function on osteoblastic differentiation in these studies remains unclear; however, variations in downstream effectors activated by AMPK under different conditions may be a contributing factor. 
Liraglutide has recently been demonstrated to prevent the osteoblastic differentiation of human vascular smooth muscle cells, which is reported to slow the process of arterial calcification $(10,11)$. As arterial calcification is considered to be similar to bone formation, this observation indicated that liraglutide may be implicated in the osteoblastic differentiation of osteoblasts. Furthermore, AMPK/mammalian target of rapamycin (mTOR) signaling cascades are involved in the mechanisms that mediate the antidiabetic effects of liraglutide (12). Thus, it is hypothesized that liraglutide is involved in osteoblastic differentiation by modulating AMPK/mTOR signaling.

Alkaline phosphatase (Alp) is responsible for hydrolyzing pyrophosphate in osteoblasts to generate inorganic phosphate, an essential component of the mineralized matrix of osteoblasts (13), while mature osteoblasts are typically characterized by high osteocalcin (OC) expression (5). OC, a structural protein of bone, has a high affinity for hydroxyapatite and regulates the generation of osteoblast mineralized matrix $(14,15)$. Due to the reciprocal association between Alp/OC expression and osteoblastic differentiation (16), Alp and $\mathrm{OC}$ are commonly used as osteoblastic differentiation markers. Mineralized matrix formation in osteoblasts is an important process during osteoblastic differentiation and is associated with the deposition of calcium phosphate salts $(14,17)$. Therefore, examination of the calcium phosphate salt content in osteoblasts is another marker of the extent of osteoblastic differentiation. Matrix mineralization can be assessed via Alizarin red S staining, which detects both microcrystalline and noncrystalline calcium phosphate salts in osteoblasts (18).

The present study aimed to investigate the effects of liraglutide treatment on osteoblastic differentiation of MC3T3-E1 cells, a clonal osteogenic cell line that maintains characteristics of osteoblasts, and is frequently used to investigate osteoblastic differentiation and function in vitro (19), using Alp, OC and mineralized matrix formation as markers of osteoblastic differentiation.

\section{Materials and methods}

Reagents and cell treatments. Compound C (AMPK inhibitor) and MHY1485 (mTOR activator) were purchased from Calbiochem(EMD Millipore, Billerica, MA, USA) and Selleck Chemicals (Shanghai, China), respectively. Rabbit anti-Alp antibody (1:10,000; catalog no. ab190931; Abcam, Cambridge, UK), mouse anti-OC antibody (1:400; catalog no. sc-74495; Santa Cruz Biotechnology, Inc., Dallas, TX, USA), rabbit anti-AMPK antibody (1:1,000; catalog no. ab32047; Abcam), rabbit anti-phosphorylated (p)-AMPK antibody (Thr172; 1:1,000; catalog no. ab133448; Abcam), rabbit anti-mTOR antibody (1:1,000; catalog no. 2972; CST Biological Reagents Co., Ltd., Shanghai, China), rabbit anti-p-mTOR antibody (Ser2448; 1:1,000; catalog no. 2971; CST Biological Reagents Co., Ltd.), rabbit anti-transforming growth factor- $\beta$ (TGF- $\beta$ ) antibody (1:500; catalog no. bs-0103R; BIOSS, Beijing, China) and mouse anti-glyceraldehyde- 3 phosphate dehydrogenase (GAPDH) antibody (1:500; catalog no. sc-365062; Santa Cruz Biotechnology) were purchased for western blotting and immunocytochemistry.
MC3T3-E1 cells, obtained from the American Type Culture Collection (Manassas, VA, USA), were maintained in Dulbecco's modified Eagle's medium (DMEM, Hyclone; GE Healthcare Life Sciences, Logan, UT, USA) supplemented with $10 \%$ fetal bovine serum (FBS, Hyclone; GE Healthcare Life Sciences), $100 \mathrm{U} / \mathrm{ml}$ penicillin and $100 \mathrm{mg} / \mathrm{ml}$ streptomycin at $37^{\circ} \mathrm{C}$ in a humidified atmosphere of $5 \% \mathrm{CO}_{2}$. Having reached $70 \%$ confluence, the culture medium was switched to commercial osteogenic differentiation medium (catalog no. MUCMX-90021; Cyagen Biotechnology Co., Ltd., Taicang, China). MC3T3-E1 cells were cultured in the osteogenic differentiation medium for 14 days, following by culture in DMEM supplemented with varying concentrations of liraglutide (catalog no. HY-P0014; MedChem Express, Shanghai, China) for a further 14 days. MC3T3-E1 cells treated with $4 \mathrm{nM}$ liraglutide were cultured in the presence or absence of Compound C or MHY1485. MC3T3-E1 cells maintained in DMEM for 28 days in the absence of any treatment were used as the negative control (NC); cells cultured in commercial osteogenic differentiation medium for 14 days and in DMEM without liraglutide for an additional 14 days were used as the positive control (PC).

Western blotting. Cells were lysed with NP-40 buffer (1\% NP-40, $0.15 \mathrm{M} \mathrm{NaCl}, 50 \mathrm{mM}$ Tris, $\mathrm{pH}$ 8.0) containing phosphatase inhibitors (okadaic acid; catalog no. ab120375; Abcam). Protein concentration was measured using a bicinchoninic acid protein assay kit (Beyotime Institute of Biotechnology, Shanghai, China). Equal amounts of protein $(20 \mu \mathrm{g})$ were separated by sodium dodecyl sulfate-polyacrylamide gel electrophoresis on $12 \%$ gels and transferred onto nitrocellulose membranes. After blocking with 5\% non-fat milk in Tris-buffered saline containing Tween (TBST) for $1 \mathrm{~h}$, membranes were incubated with primary antibody in TBST overnight at $4^{\circ} \mathrm{C}$ and subsequently incubated with anti-mouse (catalog no. A9309; Sigma-Aldrich; Merck Millipore, Darmstadt, Germany) or anti-rabbit (catalog no. ab97051; Abcam) horse radish peroxidase-conjugated secondary antibodies at a 1:2,000 dilution for $2 \mathrm{~h}$ at room temperature. Reactive proteins were detected using Pierce Enhanced Chemiluminscent and SuperSignal ${ }^{\mathrm{TM}}$ Chemiluminescent substrates (Thermo Fisher Scientific, Inc., Waltham, MA, USA). To control for loading efficiency, the blots were stripped with Mild Stripping Buffer (Abcam) at room temperature for 5-10 min and reprobed with GAPDH antibody (1:600).

Immunocytochemistry (ICC). The cells were fixed by $4 \%$ paraformaldehyde at room temperature for $10 \mathrm{~min}$ and methanol at $-20^{\circ} \mathrm{C}$ for $20 \mathrm{~min}$. Normal goat serum (10\%; Hyclone; GE Healthcare Life Sciences) was added to cells for $30 \mathrm{~min}$ to block nonspecific binding sites. The fixed cells were immunostained with primary antibodies targeting Alp (1:300) and OC (1:200) overnight at $4^{\circ} \mathrm{C}$ and the Alexa Fluor 488-conjugated goat anti-rabbit secondary antibody (1:500; catalog no. SP-9000; ZSGB-BIO, Beijing, China) for $1 \mathrm{~h}$ at $37^{\circ} \mathrm{C}$. Images were acquired with a high-resolution CoolSNAP ${ }^{\mathrm{TM}}$ CCD camera (Photometrics Inc., Tucson, AZ, USA) under the control of a computer using Leica FW4000 software version 1.2 (Leica Microsystems, Ltd., Milton Keynes, UK). 

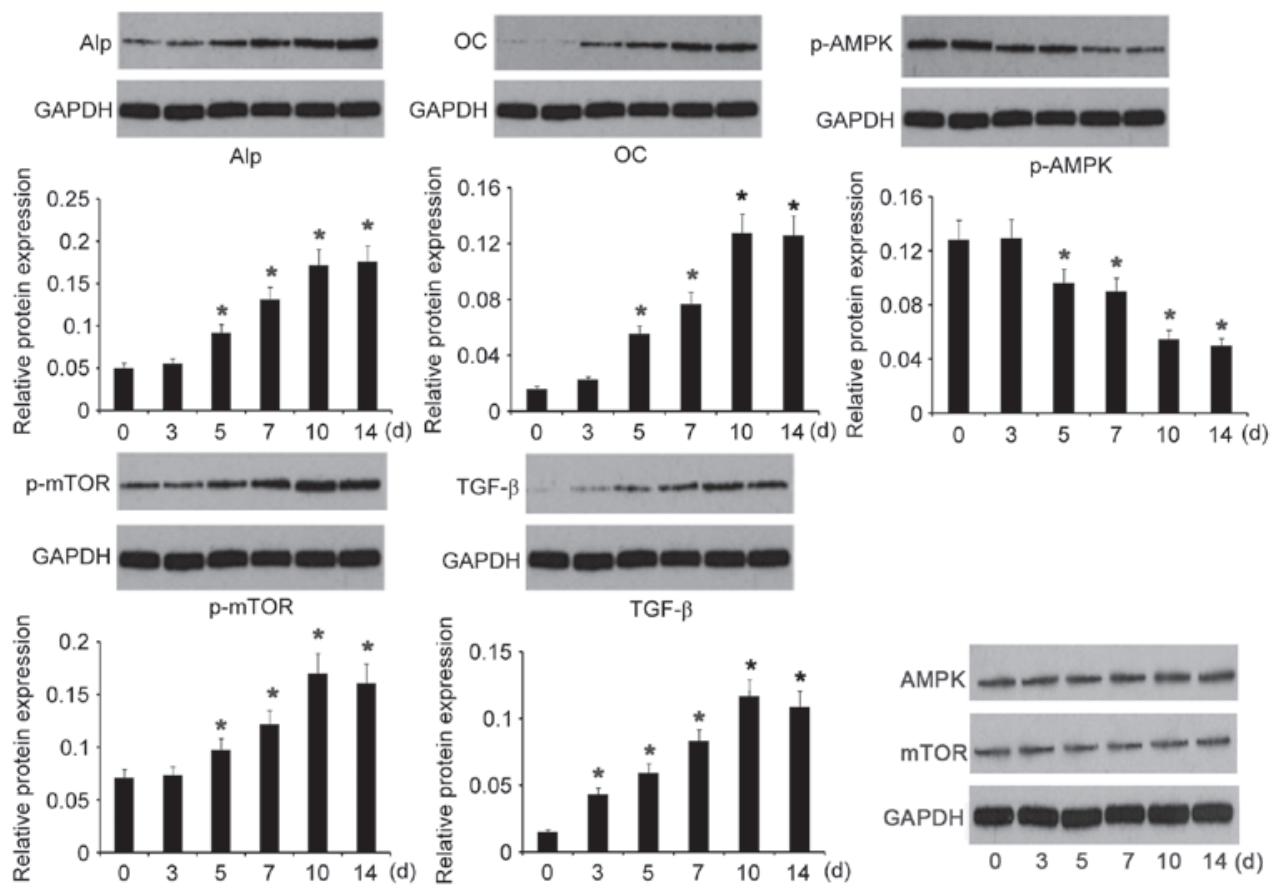

Figure 1. Relative protein expression of Alp, OC, p-AMPK, p-mTOR and TGF- $\beta$ during osteogenic differentiation. Commercial osteogenic differentiation medium was used to induce osteoblastic differentiation of MC3T3-E1 cells, and the relative protein expression of indicated proteins was analyzed by western blotting on days 0,3, 5, 7 and 14. Quantification was relative to GAPDH. Data are presented as the mean \pm standard error $(n=5)$. "P<0.05 vs. day 0 . Alp, alkaline phosphatase; OC, osteocalcin; p-AMPK, phosphorylated AMPK; p-mTOR, phosphorylated mTOR; TGF- $\beta$, transforming growth factor- $\beta$; GAPDH, glyceraldehyde 3-phosphate dehydrogenase.

Mineralization assay. The extent of matrix mineralization of MC3T3-E1 cells were determined by Alizarin Red S staining. Cells were fixed with $2 \%$ formaldehyde for $10 \mathrm{~min}$ at room temperature, followed by exposure to $2 \%$ Alizarin Red S for $40 \mathrm{~min}$. Cells were then washed with phosphate-buffered saline to remove excess dye, and cells were examined under a BA410 Optical Microscope plus Absorbance Analyzer (Motic Medical Diagnostic Systems Co., Ltd., Xiamen, China). Absorbance was measured at $550 \mathrm{~nm}$. The mineralization values were normalized to the relative value of the NC.

Statistical analysis. Data were analyzed using SPSS 12.0 software (SPSS, Inc., Chicago, IL, USA). One-way analysis of variance followed by Scheffe's post-hoc test was used for multiple comparisons between each group. $\mathrm{P}<0.05$ was considered to indicate a statistically significant difference.

\section{Results}

Osteoblastic differentiation of MC3T3-E1 cells is induced by commercial osteogenic differentiation medium. Commercial osteogenic differentiation medium was used to induce the differentiation of osteoblastic MC3T3-E1 cells towards mature osteocytes. The protein expression levels of two crucial osteoblastic differentiation markers, Alp and OC, were evaluated on days $0,3,5,7$, and 14 of incubation in osteogenic differentiation medium by western blotting. Protein expression levels of Alp and OC gradually increased during the time course, with $\sim 3$ - and 4-fold higher expression on day 14 compared with day 0 , respectively $(\mathrm{P}=0.024$ and $\mathrm{P}=0.027$; Fig. 1$)$, indicating a trend of osteoblastic MC3T3-E1 cell differentiation to mature osteocytes over this time period. In parallel with the osteoblastic differentiation of MC3T3-E1 cells, p-AMPK expression levels progressively decreased over the same period, with the lowest expression levels at 14 days $(\mathrm{P}=0.036$ vs. day 0 ). However, protein expression levels of p-mTOR and TGF- $\beta$ gradually increased over the time course. Expression levels were significantly increased on day 14 compared with day $0,(\mathrm{P}=0.039$ and $\mathrm{P}=0.017$, respectively). No significant differences were observed in AMPK and mTOR expression levels (Fig. 1). These data suggested a close association between osteoblastic differentiation of MC3T3-E1 cells and p-AMPK/p-mTOR/TGF- $\beta$ expression levels.

Increased expression of Alp and OC on day 14 compared with day 0 was also observed by ICC (Fig. 2A). Alizarin red $\mathrm{S}$ staining was used to evaluate mineral matrix formation in osteoblasts. Increased intensity of Alizarin red S staining, as determined by spectrophotometric readings, were observed over the time-course of 14 days (Fig. 2B). Notably, optical intensity was significantly increased on day 14 compared with day $0(\mathrm{P}=0.008)$. These data indicated an increased mineral matrix formation over this time period. Osteoblastic differentiation of MC3T3-E1 cells was, therefore, induced by the commercial osteogenic differentiation medium.

Liraglutide dose-dependently attenuates the osteoblastic differentiation of osteoblasts. MC3T3-E1 cells cultured in commercial osteogenic differentiation medium for 14 days, were transferred into basal culture medium and maintained for an additional 14 days in various concentrations of liraglutide $(0,0.1,0.5,1,2$ and $4 \mathrm{nM})$. Protein expression levels of Alp and $\mathrm{OC}$ were analyzed by western blotting, revealing that lira- 
A
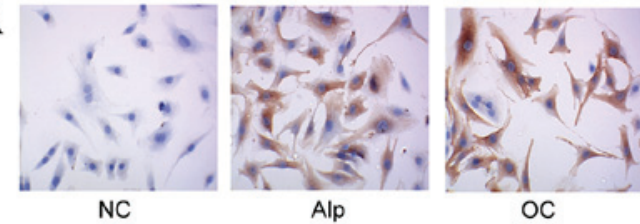

B

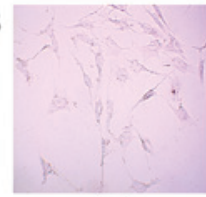

$0 \mathrm{~d}$

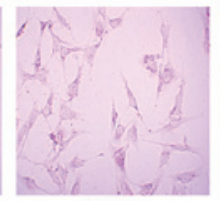

$3 d$

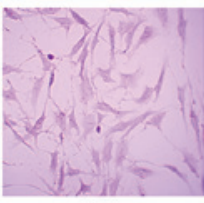

$5 \mathrm{~d}$

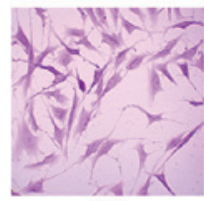

$7 d$

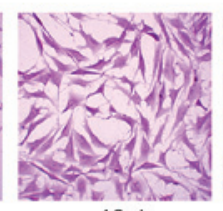

$10 \mathrm{~d}$
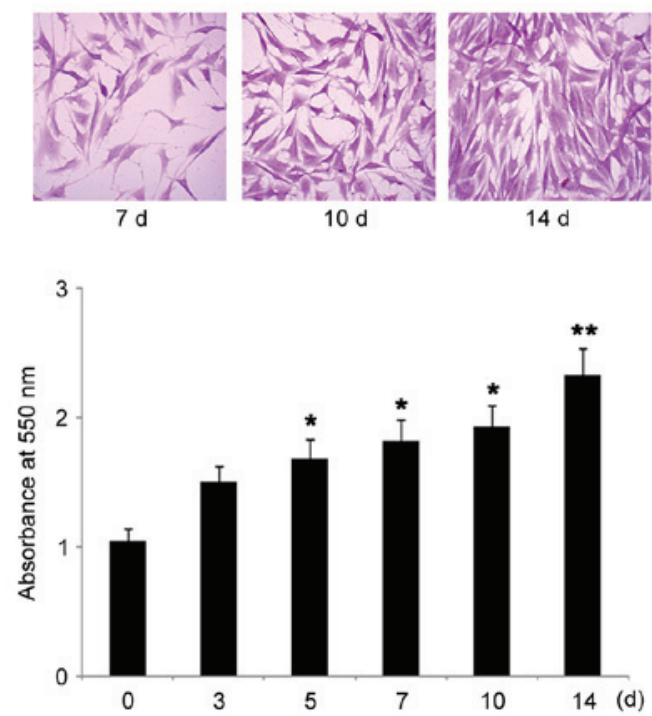

Figure 2. ICC and Alizarin Red S staining during osteogenic differentiation. Commercial osteogenic differentiation medium was used to induce the osteoblastic differentiation of MC3T3-E1 cells. (A) ICC was performed on day 14 to detect Alp and OC. Negative control cells were not stained for Alp and OC. Magnification, x400. (B) Alizarin Red S staining was performed on days $0,3,5,7$ and 14 to determine the extent of matrix mineralization Magnification, $x 200$. Data are presented as the mean \pm standard error $(n=10)$ ${ }^{*} \mathrm{P}<0.05$ and ${ }^{* *} \mathrm{P}<0.01$ vs. day 0 . ICC, immunocytochemistry; NC, negative control; Alp, alkaline phosphatase; OC, osteocalcin.

glutide dose-dependently decreased protein expression levels of Alp and OC compared with untreated PC cells; treatment with $4 \mathrm{~nm}$ liraglutide resulted in $\sim 1 / 3$ of the protein expression levels of both Alp and OC compared with untreated PC cells $(\mathrm{P}=0.035$ and $\mathrm{P}=0.038$; Fig. $3 \mathrm{~A})$. In addition, matrix mineralization of osteoblasts was attenuated by liraglutide in a dose-dependent manner, as determined by Alizarin red S staining (Fig. 3B). Liraglutide at a concentration of $4 \mathrm{nM}$ decreased the absorbance to the lowest level of all treatment groups ( $\mathrm{P}=0.009$ vs. $\mathrm{PC}$ group). These results indicated that liraglutide may inhibit and/or reverse osteoblastic differentiation.

AMPK/mTOR signaling is involved in the inhibitory effect of liraglutide on osteoblastic differentiation. MC3T3-E1 cells cultured in commercial osteogenic differentiation medium for 14 days, followed by a further 14 days culturing in basal culture medium with $4 \mathrm{nM}$ liraglutide exhibited significantly lower formation of mineralized matrix compared with untreated PC cells ( $\mathrm{P}=0.009$; Fig. 4A) and significantly reduced protein expression levels of Alp $(\mathrm{P}=0.035$; Fig. 4B) and $\mathrm{OC}(\mathrm{P}=0.038$; Fig. 4B). Liraglutide treatment significantly increased p-AMPK protein expression levels compared with untreated PC cells $(P=0.041)$ whereas the levels of $p$-mTOR and TGF- $\beta$ were significantly reduced compared with PC $(\mathrm{P}=0.041$ and $\mathrm{P}=0.035$, respectively). However, co-treatment of $4 \mathrm{nM}$ liraglutide and $10 \mu \mathrm{M}$ Compound $\mathrm{C}$, an inhibitor of AMPK, prevented the liraglutide-induced increase in p-AMPK levels compared with liraglutide treated cells, and restored the levels of Alp, OC, p-mTOR and TGF- $\beta$ (Fig. 4B), and matrix mineralization (Fig. 4A) to levels comparable with positive control cells. Similarly, co-treatment with $4 \mathrm{nM}$ liraglutide and $1 \mu \mathrm{M}$ MHY1485, an mTOR activator, resulted in protein expression levels of Alp, OC, p-mTOR and TGF- $\beta$ (Fig. 4B) and matrix mineralization (Fig. 4A) comparable to those of positive control cells cells. However, MHY1485 did not similarly attenuate the liraglutide-induced increase in p-AMPK levels, resulting in significantly increased protein expression levels of $\mathrm{p}$-AMPK compared with positive control cells $(\mathrm{P}=0.04$; Fig. 4B). Protein expression levels of AMPK and $\mathrm{mTOR}$ were unaffected by liraglutide and the AMPK and mTOR inhibitors. These data suggested that liraglutide affects the activities of AMPK and mTOR but not their expression, and that the AMPK/mTOR axis is potentially involved in the effect of liraglutide on osteoblastic differentiation.

\section{Discussion}

The present study demonstrated that liraglutide attenuated osteoblastic differentiation, based on the decreased protein levels of Alp and OC resulting from liraglutide treatment, and prevention of mineralized matrix formation in osteoblast MC3T3-E1 cells following exposure to liraglutide. Protein expression levels of Alp and OC in osteoblasts were decreased by liraglutide in a dose-dependent manner, indicating that liraglutide inhibits differentiation of osteoblast MC3T3-E1 cells. Liraglutide also dose-dependently decreased Alizarin red S staining of MC3T3-E1 cells, indicating that liraglutide inhibits calcium phosphate salt deposition, resulting in diminished osteoblastic differentiation of MC3T3-E1 cells.

Since previous studies have suggested that AMPK signaling may be involved in both osteoblastic differentiation and other biological functions of liraglutide $(6-9,12)$, the present study investigated AMPK signaling to provide insights into the adverse effects of liraglutide on osteoblastic differentiation. AMPK activity, assessed through detection of phosphorylated AMPK, was decreased during osteoblastic differentiation of MC3T3-E1 cells induced by commercial osteogenic differentiation medium. However, when such differentiation was inhibited using liraglutide, AMPK was activated at similar levels as in undifferentiated cells. Furthermore, inhibition of AMPK signaling through treatment with compound C abolished the inhibitory effect of liraglutide on osteoblast differentiation. These data, therefore, support the hypothesis that AMPK signaling serves a critical role in the liraglutide-associated attenuation of osteoblastic differentiation.

AMPK signaling has been previously demonstrated to attenuate or enhance osteoblastic differentiation, according to the manner in which AMPK is activated and which downstream targets of AMPK are regulated (6-9). mTOR, an important 
A
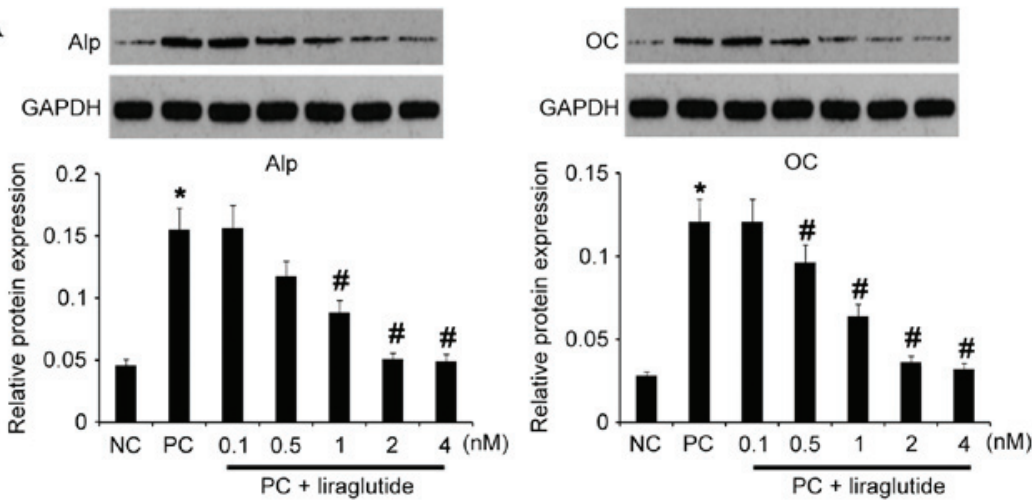

B

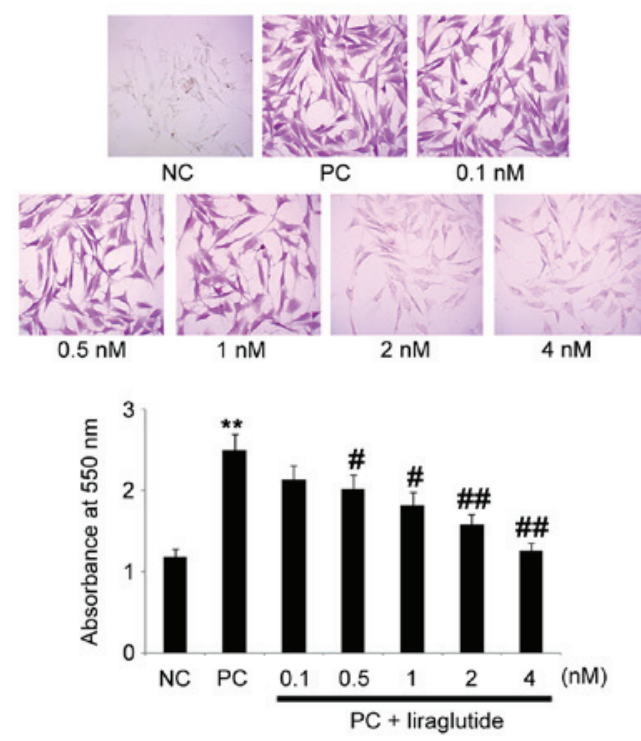

Figure 3. Effects of liraglutide treatment on Alp and OC protein expression levels, and matrix mineralization. Following culture in commercial osteogenic differentiation medium for 14 days, MC3T3-E1 cells were cultured for an additional 14 days in basal culture medium with various concentrations of liraglutide $(0,0.1,0.5,1,2$ and $4 \mathrm{nM})$. MC3T3-E1 cells maintained in DMEM for 28 days with no treatment were used as the NC; cells cultured in the commercial osteogenic differentiation medium for 14 days and in DMEM medium without liraglutide for a further 14 days were used as the PC. (A) Relative protein expression of Alp and OC was analyzed by western blot and quantified relative to GAPDH $(\mathrm{n}=5)$. (B) Matrix mineralization was determined by Alizarin Red S staining $(\mathrm{n}=10)$. Magnification, $\mathrm{x} 200$. Data are presented as the mean \pm standard error. ${ }^{*} \mathrm{P}<0.05,{ }^{* *} \mathrm{P}<0.05 \mathrm{vs}$. NC; ${ }^{\#} \mathrm{P}<0.05,{ }^{\# \#} \mathrm{P}<0.01$ vs. PC. Alp, alkaline phosphatase; GAPDH, glyceraldehyde 3-phosphate dehydrogenase; NC, negative control; PC, positive control; OC, osteocalcin.

down-stream target of AMPK, stimulates osteoblastic differentiation $(10,20)$. The present study demonstrated that mTOR phosphorylation is negatively associated with that of AMPK, but differentiation of MC3T3-E1 osteoblasts is functionally associated with increased mTOR activity. Importantly, both the AMPK inhibitor and mTOR activator restored mTOR activity (mTOR phosphorylation) and abolished the inhibitory effect of liraglutide on osteoblast differentiation. However, the mTOR activator had no effect on the levels of p-AMPK, indicating that liraglutide attenuates osteoblastic differentiation of MC3T3-E1 cells via AMPK-mediated mTOR suppression.

TGF- $\beta$ is the most abundant cytokine in bone, with a key role in osteoblastic differentiation $(21,22)$. To initiate cellular responses TGF- $\beta$ receptors are required to transduce the TGF- $\beta$ signal and activate intracellular transcription factors (23). Osteoblasts have been demonstrated to secrete TGF- $\beta$, and to express a large variety of high affinity TGF- $\beta$ receptors, suggesting that TGF- $\beta$ acts as an autocrine and paracrine cytokine, regulating multiple physiological processes in osteoblasts $(15,21)$. Although the effect of TGF- $\beta$ on the regulation of osteoblastic differentiation remains unclear, previous studies have demonstrated that TGF- $\beta$ promotes early differentiation of osteoblasts $(24,25)$. The present study revealed that protein expression levels of TGF- $\beta$ followed similar trends to p-mTOR, and that both the AMPK inhibitor and mTOR activator abolished the inhibitory effect of liraglutide on osteoblast differentiation. Previous studies have demonstrated that ribosomal protein S6 kinase B1, a well-characterized downstream target of mTOR, regulates the functions and synthesis of multiple cytokines to modulate osteogenesis, including platelet-derived growth factor, fibroblast growth factor and vascular endothelial growth factor (26). TGF- $\beta$ is, therefore, speculated to mediate the effects of mTOR on osteoblastic differentiation.

The clinical implications of liraglutide attenuation of osteoblastic differentiation remain unknown. Attenuated osteoblastic differentiation inhibits bone formation, which may, therefore, increase the risk of osteoporosis and bone 
A
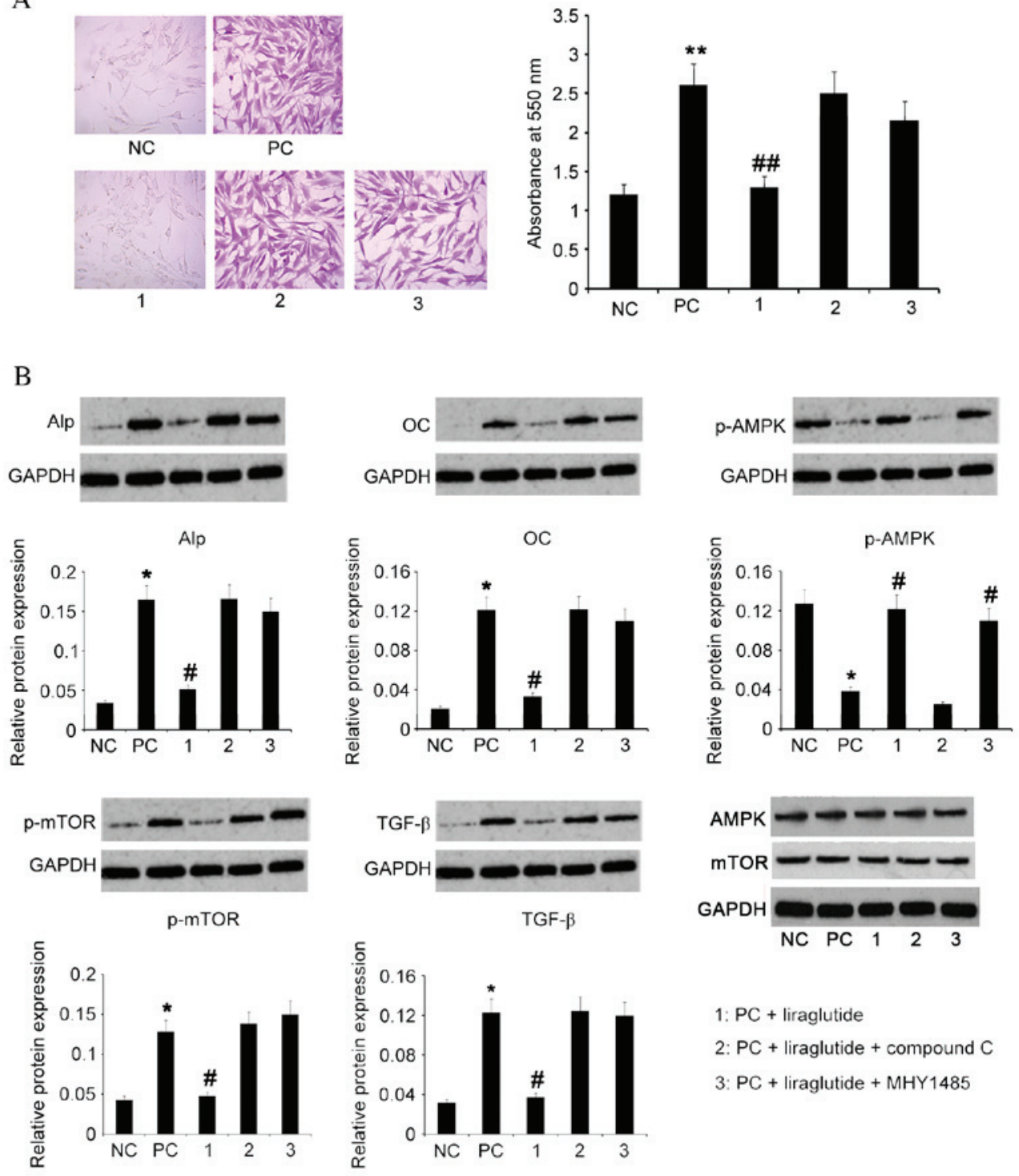

Figure 4. Effects of liraglutide treatments and AMPK inhibitor/mTOR activator on matrix mineralization and relative protein levels of Alp, OC, p-AMPK, p-mTOR and TGF- $\beta$. Following culture in commercial osteogenic differentiation medium for 14 days, MC3T3-E1 cells were cultured for a further 14 days in basal culture medium with $4 \mathrm{nM}$ liraglutide, and $4 \mathrm{nM}$ liraglutide plus AMPK inhibitor, $10 \mu \mathrm{M}$ compound C, or $1 \mu \mathrm{M}$ mTOR activator, MHY1485. MC3T3-E1 cells maintained in DMEM for 28 days with no treatment were used as the NC; cells cultured in the commercial osteogenic differentiation medium for 14 days and in DMEM without liraglutide for a further 14 days were used as the PC. (A) Matrix mineralization was determined by Alizarin Red S staining $(\mathrm{n}=10)$. Magnification, $\mathrm{x} 200$. (B) Relative protein expression was analyzed by western blot and quantified relative to GAPDH $(\mathrm{n}=5)$. Data are presented as the mean \pm standard error. Bars with letters means they significantly differ with positive control $(\mathrm{P}<0.05)$. ${ }^{*} \mathrm{P}<0.05,{ }^{* *} \mathrm{P}<0.05 \mathrm{vs}$. NC; ${ }^{*} \mathrm{P}<0.05$, ${ }^{\# \#} \mathrm{P}<0.01 \mathrm{vs}$. $\mathrm{PC}$. NC, negative control; PC, positive control; Alp, alkaline phosphatase; OC, osteocalcin; p-, phosphorylated; AMPK, adenosine monophosphate-activated protein kinase; mTOR, mechanistic target of rapamycin; TGF- $\beta$, transforming growth factor- $\beta$; GAPDH, glyceraldehyde 3-phosphate dehydrogenase.

fractures (27). However, the liraglutide-induced reduction in endogenous TGF- $\beta$ expression demonstrated in the present study may inhibit the generation of osteophytes, as demonstrated in previous in vivo studies $(28,29)$. Additionally, as osteoblastic differentiation is associated with calcium and phosphorus metabolism (26), it is possible that negative regulation of osteoblastic differentiation by liraglutide may also impact calcium and phosphorus metabolism.

In summary, the present study demonstrated that liraglutide attenuated osteoblastic differentiation and inhibited the expression of osteoblastic differentiation markers, Alp and OC, and the formation of mineralized matrix in osteoblasts. The protein expression level of p-AMPK was significantly increased when osteoblastic differentiation of MC3T3-E1 cells was attenuated by liraglutide, whereas levels of p-mTOR and TGF- $\beta$ were decreased under the same conditions. An AMPK inhibitor and mTOR activator both attenuated the inhibitory effect of liraglutide on osteoblastic differentiation and restored p-mTOR protein levels to those of untreated cells. However, the mTOR activator did not restore p-AMPK protein expression levels to that of untreated cells. Thus, the present study demonstrated that liraglutide attenuated osteoblastic differentiation of MC3T3-E1 cells via modulation of AMPK/mTOR signaling. The present study revealed a novel function of liraglutide, which contributes to the understanding of its pharmacological and physiological effects in clinical settings. 


\section{Acknowledgements}

This study was partially funded by the National Natural Science Foundation of China (grant no. 81271940) and the Project of Furong Scholar of Hunan Province.

\section{References}

1. Jendle J,Nauck MA, Matthews DR, Frid A,Hermansen K, Düring M, Zdravkovic M, Strauss BJ and Garber AJ; LEAD-2 and LEAD-3 Study Groups: Weight loss with liraglutide, a once-daily human glucagon-like peptide-1 analogue for type 2 diabetes treatment as monotherapy or added to metformin, is primarily as a result of a reduction in fat tissue. Diabetes Obes Metab 11: 1163-1172, 2009.

2. Zhao X, Liu G, Shen H, Gao B, Li X, Fu J, Zhou J and Ji Q: Liraglutide inhibits autophagy and apoptosis induced by high glucose through GLP-1R in renal tubular epithelial cells. Int $\mathbf{J}$ Mol Med 35: 684-692, 2015.

3. Shyangdan D, Cummins E, Royle P and Waugh N: Liraglutide for the treatment of type 2 diabetes. Health Technol Assess 15 (Suppl 1): S77-S86, 2011.

4. Gough SC: Liraglutide: From clinical trials to clinical practice. Diabetes Obes Metab 14 (Suppl 2): S33-S40, 2012.

5. Lind T, Sundqvist A, Hu L, Pejler G, Andersson G, Jacobson A and Melhus H: Vitamin a is a negative regulator of osteoblast mineralization. PLoS One 8: e82388, 2013.

6. Kanazawa I, Yamaguchi T, Yano S, Yamauchi M, Yamamoto M and Sugimoto T: Adiponectin and AMP kinase activator stimulate proliferation, differentiation, and mineralization of osteoblastic MC3T3-E1 cells. BMC Cell Biol 8: 51, 2007.

7. Zhong X, Xiu LL, Wei GH, Liu YY, Su L, Cao XP, Li YB and Xiao HP: Bezafibrate enhances proliferation and differentiation of osteoblastic MC3T3-E1 cells via AMPK and eNOS activation. Acta Pharmacol Sin 32: 591-600, 2011.

8. Kim JY, Min JY, Baek JM, Ahn SJ, Jun HY, Yoon KH, Choi MK, Lee MS and Oh J: CTRP3 acts as a negative regulator of osteoclastogenesis through AMPK-c-Fos-NFATc1 signaling in vitro and RANKL-induced calvarial bone destruction in vivo. Bone 79: 242-251, 2015.

9. Wei J, Shimazu J, Makinistoglu MP, Maurizi A, Kajimura D, Zong H, Takarada T, Iezaki T, Pessin JE, Hinoi E and Karsenty G: Glucose uptake and Runx2 synergize to orchestrate osteoblast differentiation and bone formation. Cell 161: 1576-1591, 2015.

10. Zhan JK, Wang YJ, Wang Y, Tang ZY, Tan P, Huang W and Liu YS: Adiponectin attenuates the osteoblastic differentiation of vascular smooth muscle cells through the AMPK/mTOR pathway. Exp Cell Res 323: 352-358, 2014.

11. Zhan JK, Wang YJ, Wang Y, Tang ZY, Tan P, Huang W and Liu YS: The protective effect of GLP-1 analogue in arterial calcification through attenuating osteoblastic differentiation of human VSMCs. Int J Cardiol 189: 188-193, 2015

12. Miao XY, Gu ZY, Liu P, Hu Y, Li L, Gong YP, Shu H, Liu Y and Li CL: The human glucagon-like peptide-1 analogue liraglutide regulates pancreatic beta-cell proliferation and apoptosis via an AMPK/mTOR/P70S6K signaling pathway. Peptides 39: 71-79, 2013.

13. de la Croix Ndong J, Makowski AJ, Uppuganti S, Vignaux G, Ono K, Perrien DS, Joubert S, Baglio SR, Granchi D, Stevenson DA, et al: Corrigendum: Asfotase- $\alpha$ improves bone growth, mineralization and strength in mouse models of neurofibromatosis type-1. Nat Med 21: 414, 2015.
14. D'Alonzo RC, Kowalski AJ, Denhardt DT, Nickols GA and Partridge NC: Regulation of collagenase-3 and osteocalcin gene expression by collagen and osteopontin in differentiating MC3T3-E1 cells. J Biol Chem 277: 24788-24798, 2002.

15. Hughes-Fulford M and Li CF: The role of FGF-2 and BMP-2 in regulation of gene induction, cell proliferation and mineralization. J Orthop Surg Res 6: 8, 2011.

16. Owen TA, Aronow M, Shalhoub V, Barone LM, Wilming L, Tassinari MS, Kennedy MB, Pockwinse S, Lian JB and Stein GS: Progressive development of the rat osteoblast phenotype in vitro: Reciprocal relationships in expression of genes associated with osteoblast proliferation and differentiation during formation of the bone extracellular matrix. J Cell Physiol 143: 420-430, 1990.

17. Yang L, Cheng P, Chen C, He HB, Xie GQ, Zhou HD, Xie H, Wu XP and Luo XH: miR-93/Sp7 function loop mediates osteoblast mineralization. J Bone Miner Res 27: 1598-1606, 2012.

18. Paul H, Reginato AJ and Schumacher HR: Alizarin red S staining as a screening test to detect calcium compounds in synovial fluid. Arthritis Rheum 26: 191-200, 1983.

19. Sudo H, Kodama HA, Amagai Y, Yamamoto S and Kasai S: In vitro differentiation and calcification in a new clonal osteogenic cell line derived from newborn mouse calvaria. J Cell Biol 96: 191-198, 1983

20. Singha UK, Jiang Y, Yu S, Luo M, Lu Y, Zhang J and Xiao G Rapamycin inhibits osteoblast proliferation and differentiation in MC3T3-E1 cells and primary mouse bone marrow stromal cells. J Cell Biochem 103: 434-446, 2008.

21. Ehnert S, Baur J, Schmitt A, Neumaier M, Lucke M, Dooley S, Vester H, Wildemann B, Stöckle U and Nussler AK: TGF- $\beta 1$ as possible link between loss of bone mineral density and chronic inflammation. PLoS One 5: e14073, 2010.

22. Janssens K, ten Dijke P, Janssens S and Van Hul W: Transforming growth factor-betal to the bone. Endocr Rev 26: 743-774, 2005.

23. Latella G, Vetuschi A, Sferra R, Speca S and Gaudio E Localization of $\alpha v \beta 6$ integrin-TGF- $\beta 1 / \mathrm{Smad} 3, \mathrm{mTOR}$ and PPAR $\gamma$ in experimental colorectal fibrosis. Eur J Histochem 57: e40, 2013.

24. Manzano-Moreno FJ, Medina-Huertas R, Ramos-Torrecillas J, García-Martínez O and Ruiz C: The effect of low-level diode laser therapy on early differentiation of osteoblast via BMP-2/TGF- $\beta 1$ and its receptors. J Craniomaxillofac Surg 43: 1926-1932, 2015.

25. Suzuki E, Ochiai-Shino H, Aoki H, Onodera S, Saito A, Saito A and Azuma T: Akt activation is required for TGF- $\beta 1$-induced osteoblast differentiation of MC3T3-E1 pre-osteoblasts. PLoS One 9: e112566, 2014.

26. Xiang X, Zhao J, Xu G, Li Y and Zhang W: mTOR and the differentiation of mesenchymal stem cells. Acta Biochim Biophys Sin (Shanghai) 43: 501-510, 2011.

27. Wanachewin O, Boonmaleerat K, Pothacharoen P, Reutrakul V and Kongtawelert P: Sesamin stimulates osteoblast differentiation through $\mathrm{p} 38$ and ERK1/2 MAPK signaling pathways. BMC Complement Altern Med 12: 71, 2012.

28. Scharstuhl A, Glansbeek HL, van Beuningen HM, Vitters EL, van der Kraan PM and van den Berg WB: Inhibition of endogenous TGF-beta during experimental osteoarthritis prevents osteophyte formation and impairs cartilage repair. J Immunol 169: 507-514, 2002.

29. Scharstuhl A, Vitters EL, van der Kraan PM and van den Berg WB: Reduction of osteophyte formation and synovial thickening by adenoviral overexpression of transforming growth factor beta/bone morphogenetic protein inhibitors during experimental osteoarthritis. Arthritis Rheum 48: 3442-3451, 2003. 